\title{
ON CERTAIN LATTICES ASSOCIATED WITH GENERIC DIVISION ALGEBRAS
}

\author{
NICOLE LEMIRE AND MARTIN LORENZ
}

\begin{abstract}
Let $\mathcal{S}_{n}$ denote the symmetric group on $n$ letters. We consider the $\mathcal{S}_{n}$-lattice $A_{n-1}=\left\{\left(z_{1}, \ldots, z_{n}\right) \in \mathbb{Z}^{n} \mid \sum_{i} z_{i}=0\right\}$, where $\mathcal{S}_{n}$ acts on $\mathbb{Z}^{n}$ by permuting the coordinates, and its squares $A_{n-1}^{\otimes 2}$, Sym ${ }^{2} A_{n-1}$, and $\bigwedge^{2} A_{n-1}$. For odd values of $n$, we show that $A_{n-1}^{\otimes 2}$ is equivalent to $\bigwedge^{2} A_{n-1}$ in the sense of ColliotThélène and Sansuc [6]. Consequently, the rationality problem for generic division algebras amounts to proving stable rationality of the multiplicative invariant field $k\left(\bigwedge^{2} A_{n-1}\right)^{\mathcal{S}_{n}}$ ( $n$ odd). Furthermore, confirming a conjecture of Le Bruyn [16], we show that $n=2$ and $n=3$ are the only cases where $A_{n-1}^{\otimes 2}$ is equivalent to a permutation $\mathcal{S}_{n}$-lattice. In the course of the proof of this result, we construct subgroups $\mathcal{H} \leq \mathcal{S}_{n}$, for all $n$ that are not prime, so that the multiplicative invariant algebra $k\left[A_{n-1}\right]^{\mathcal{H}}$ has a non-trivial Picard group.
\end{abstract}

\section{INTRODUCTION}

This note addresses two points raised in Le Bruyn's excellent survey article [16] on the rationality problem for the center of generic division algebras. This problem, in a reformulation due to Formanek [10], asks whether the multiplicative invariant field

$$
k\left(A_{n-1}^{\otimes 2}\right)^{\mathcal{S}_{n}}
$$

that is associated with the action of the symmetric group $\mathcal{S}_{n}$ on the tensor square of the root lattice $A_{n-1}$ is (stably) rational. Recall that $A_{n-1}=\left\{\left(z_{1}, \ldots, z_{n}\right) \in\right.$ $\left.\mathbb{Z}^{n} \mid \sum_{i} z_{i}=0\right\}$, with $\mathcal{S}_{n}$ acting on $\mathbb{Z}^{n}$ by permuting the coordinates. The lattice $A_{n-1}^{\otimes 2}=A_{n-1} \otimes A_{n-1}$ can be replaced by lattices that are equivalent in a certain specific sense - see Sections 1 and 2 for precise formulations - and are, hopefully, more tractable than $A_{n-1}^{\otimes 2}$. Indeed, most cases where the rationality problem for generic division algebras has been successfully treated rely on such a replacement. This is indicated in the following table, where $\sim$ denotes the aforementioned lattice equivalence:

1991 Mathematics Subject Classification. 16G30, 20C10, 20C30, 13A50, 16K40, 20J06.

Key words and phrases. multiplicative invariant field, (stably) rational field extension, permutation lattice, root lattice, generic division algebra, Picard group, cohomology of groups.

Research of the second author supported in part by NSF Grant DMS-9618521. 


\begin{tabular}{|c|c|}
\hline$n$ & $A_{n-1}^{\otimes 2}$ \\
\hline \hline 2 & $\sim 0$ \\
\hline 3 & $\sim 0$ \\
\hline 4 & $\sim A_{3}^{*} \otimes \mathbb{Z}^{-}$ \\
\hline 5 or 7 & $\sim A_{n-1}^{*}$ \\
\hline
\end{tabular}

Here, (. . * denotes dual lattices and $\mathbb{Z}^{-}=\bigwedge^{n} \mathbb{Z}^{n}$ is the sign lattice for $\mathcal{S}_{n}$. The case $n=2$ is obvious; in fact, $A_{1}^{\otimes 2} \cong \mathbb{Z}$ is the trivial $\mathcal{S}_{2}$-lattice. Formanek [10, [11] dealt with $n=3$ and $n=4$, the equivalence $A_{3}^{\otimes 2} \sim A_{3}^{*} \otimes \mathbb{Z}^{-}$being crucial for $n=4$. Finally, the equivalence $A_{n-1}^{\otimes 2} \sim A_{n-1}^{*}$ is the operative fact in Bessenrodt and Le Bruyn's solution of the rationality problem for $n=5$ and $n=7$ [3]. More recently, Beneish [2] has given a simpler proof of this equivalence (which is known to be false for prime values of $n>7$ [3]).

Our first contribution is in response to the "Representation Question" of [16], which asks to find $\mathcal{S}_{n}$-lattices equivalent to $A_{n-1}^{\otimes 2}$ but having lower rank. We show that, for odd values of $n$ and for $n=2$ and $n=4$, the exterior square $\bigwedge^{2} A_{n-1}$ serves that purpose, its rank being about half that of $A_{n-1}^{\otimes 2}$. (For even $n \geq 6$, however, $\bigwedge^{2} A_{n-1}$ is not equivalent to $A_{n-1}^{\otimes 2}$.) The equivalence $A_{n-1}^{\otimes 2} \sim \bigwedge^{2} A_{n-1}$ covers the cases $n=2,3,4$ in the above table, but not $n=5,7$. We do not touch here on the actual rationality problem for the invariant field $k\left(\bigwedge^{2} A_{n-1}\right)^{\mathcal{S}_{n}}$ which does seem rather formidable still. Even the case $n=4$, where $\bigwedge^{2} A_{3} \cong A_{3}^{*} \otimes \mathbb{Z}^{-}$, does require some effort; see [11] or [12]. In this connection, it is perhaps worth remarking that the rationality problem for the invariant field of the $\mathcal{S}_{4}$-lattice $A_{3} \otimes \mathbb{Z}^{-}$appears to be open; it is in fact the only case of a 3 -dimensional multiplicative invariant field whose rationality was left undecided in 12 .

As our second contribution, we confirm a conjecture of Le Bruyn [16, p. 108] by showing that $n=2$ and $n=3$ are the only cases where $A_{n-1}^{\otimes 2} \sim 0$. This was previously known for prime values of $n>3$ [3, Corollary 1(a)] and for all $n$ that are not squarefree [20]. After work on the present article was completed, Le Bruyn pointed out to us that his conjecture, expressed in terms of generic norm tori, was also proved by Cortella and Kunyavskiu [8]. The interest in faithful $\mathcal{S}_{n}$-lattices $M \sim 0$, often called quasi-permutation lattices, stems from the fact that their multiplicative invariant fields $k(M)^{\mathcal{S}_{n}}$ are known to be stably rational over $k$; see Section 1.4 below. Thus, our second result is largely negative in its implications for the rationality problem for generic division algebras. Nevertheless, it is of some interest in its own right. In fact, the core of its proof is a nontriviality result for the Picard groups of certain algebras of multiplicative invariants. Such nontrivial Picard groups, far from ubiquitous, give rise to nontrivial torsion in the Grothendieck group $G_{0}$ of certain polycyclic group algebras [18], a phenomenon in need of further elucidation; see [13], [14], 115] for work on this topic. 
In Sections 1 and 2 of this article, we have collected the requisite background material, while Sections 3 and 4 contain our main results. The latter two sections each are preceded by an introductory paragraph laying out their contents in a more complete and technical fashion.

Notations and Conventions. Throughout this note, $\mathcal{G}$ denotes a finite group, $\mathcal{S}_{n}$ is the symmetric group on $\{1, \ldots, n\}$, and $k$ will denote a commutative field. Furthermore, $\otimes=\otimes_{\mathbb{Z}}$ and $(.)^{*}=\operatorname{Hom}_{\mathbb{Z}}(., \mathbb{Z})$. If $\mathcal{H} \leq \mathcal{G}$ is a subgroup of $\mathcal{G}$ and $M$ a $\mathbb{Z}[\mathcal{H}]$-module then $M \uparrow_{\mathcal{H}}^{\mathcal{G}}=\mathbb{Z}[\mathcal{G}] \otimes_{\mathbb{Z}[\mathcal{H}]} M$ denotes the induced $\mathbb{Z}[\mathcal{G}]$-module.

\section{Equivalence of Lattices and of Fields}

1.1. $\mathcal{G}$-lattices. We recall some standard definitions. A $\mathcal{G}$-lattice is a (left) module over the integral group ring $\mathbb{Z}[\mathcal{G}]$ that is free of finite rank as $\mathbb{Z}$-module. A $\mathcal{G}$-lattice $M$ is called faithful if the structure map $\mathcal{G} \rightarrow \operatorname{Aut}_{\mathbb{Z}}(M)$ is injective. Further, $M$ is

- a permutation lattice if $M$ has a $\mathbb{Z}$-basis, say $X$, that is permuted by $\mathcal{G}$; we will write such a lattice as $M=\mathbb{Z}[X]$.

- stably permutation if $M \oplus P \cong Q$ holds for suitable permutation $\mathcal{G}$-lattices $P$ and $Q$.

- permutation projective (or invertible) if $M$ is a direct summand of some permutation $\mathcal{G}$-lattice.

- coflasque if $H^{1}(\mathcal{H}, M)=0$ holds for all subgroups $\mathcal{H} \leq \mathcal{G}$ or, equivalently, $\operatorname{Ext}_{\mathbb{Z}[\mathcal{G}]}(P, M)=0$ holds for all permutation projective $\mathcal{G}$-lattices $P$; see 21, Lemma 8.3] for the equivalence.

The above properties are successively weaker.

Lemma. Let $M, P$, and $Q$ be $\mathcal{G}$-lattices, with $P$ permutation projective and $Q$ coflasque. Suppose that there is an exact sequence

$$
0 \rightarrow M \rightarrow P \rightarrow Q / \alpha Q \rightarrow 0
$$

with $\alpha \in \operatorname{End}_{\mathbb{Z}[\mathcal{G}]}(Q)$ injective. Then there is an exact sequence of $\mathcal{G}$-lattices

$$
0 \rightarrow M \rightarrow P \oplus Q \rightarrow Q \rightarrow 0
$$


Proof. Consider the pull-back diagram

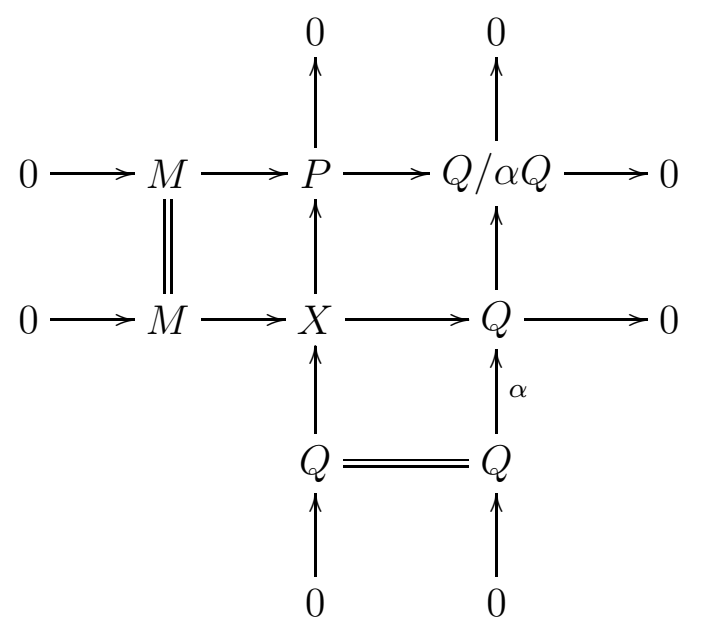

The middle column splits, by the assumptions on $P$ and $Q$, and hence the middle row yields the required sequence.

1.2. Equivalence of lattices. Following [6], two $\mathcal{G}$-lattices $M$ and $N$ are called equivalent, written $M \sim N$, provided there are exact sequences of $\mathcal{G}$-lattices

$$
0 \rightarrow M \rightarrow E \rightarrow P \rightarrow 0 \quad \text { and } \quad 0 \rightarrow N \rightarrow E \rightarrow Q \rightarrow 0
$$

where $P$ and $Q$ are permutation $\mathcal{G}$-lattices. For a direct proof that this does indeed define an equivalence relation and for further background, see [6, Lemma 8] or [21]. Lattices $M \sim 0$ are called quasi-permutation; explicitly, $M$ is quasi-permutation iff there is an exact sequence of $\mathcal{G}$-lattices $0 \rightarrow M \rightarrow P \rightarrow Q \rightarrow 0$ where $P$ and $Q$ are permutation lattices.

In the following lemma, we collect some basic properties of the equivalence $\sim$. All $M, N$ etc. are understood to be $\mathcal{G}$-lattices and all maps considered are $\mathcal{G}$-equivariant.

Lemma. (i) $M \sim N$ and $M^{\prime} \sim N^{\prime} \quad \Longrightarrow \quad M \oplus M^{\prime} \sim N \oplus N^{\prime}$.

(ii) If $0 \rightarrow M \rightarrow N \rightarrow S \rightarrow 0$ is exact with $S$ stably permutation then $M \sim N$.

Proof. (i) From (1) we obtain exact sequences $0 \rightarrow M \oplus M^{\prime} \rightarrow E \oplus M^{\prime} \rightarrow P \rightarrow 0$ and $0 \rightarrow N \oplus M^{\prime} \rightarrow E \oplus M^{\prime} \rightarrow Q \rightarrow 0$; so $M \oplus M^{\prime} \sim N \oplus M^{\prime}$. Similarly, $N \oplus M^{\prime} \sim N \oplus N^{\prime}$, and hence $M \oplus M^{\prime} \sim N \oplus N^{\prime}$, by transitivity of $\sim$.

(ii) Say $S \oplus P \cong Q$ for permutation lattices $P$ and $Q$. Then we have exact sequences $0 \rightarrow M \rightarrow N \oplus P \rightarrow S \oplus P \cong Q \rightarrow 0$ and $0 \rightarrow N \rightarrow N \oplus P \rightarrow P \rightarrow 0$, proving $M \sim N$.

1.3. $\mathcal{G}$-fields. A $\mathcal{G}$-field is a field $F$ together with a given action of $\mathcal{G}$ by automorphisms on $F$. Following [2], we say that two $\mathcal{G}$-fields $K$ and $F$ containing a common $\mathcal{G}$-subfield $k$ are stably isomorphic as $\mathcal{G}$-fields over $k$ provided, for suitable $r$ and $s$, 
there is a $\mathcal{G}$-equivariant field isomorphism

$$
K\left(x_{1}, \ldots, x_{r}\right) \stackrel{\cong}{\longrightarrow} F\left(y_{1}, \ldots, y_{s}\right)
$$

which is the identity on $k$. Here, the $x$ 's and $y$ 's are transcendental over $K$ and $F$, resp., and $K\left(x_{1}, \ldots, x_{r}\right)$ and $F\left(y_{1}, \ldots, y_{s}\right)$ are regarded as $\mathcal{G}$-fields with the trivial $\mathcal{G}$-action on the $x$ 's and $y$ 's. In particular, $K\left(x_{1}, \ldots, x_{r}\right)^{\mathcal{G}}=K^{\mathcal{G}}\left(x_{1}, \ldots, x_{r}\right)$ and similarly for $F\left(y_{1}, \ldots, y_{s}\right)$. The above isomorphism restricts to an isomorphism $K^{\mathcal{G}}\left(x_{1}, \ldots, x_{r}\right) \stackrel{\cong}{\longrightarrow} F^{\mathcal{G}}\left(y_{1}, \ldots, y_{s}\right)$ which is the identity on $k^{\mathcal{G}}$; so:

If $K$ and $F$ are stably isomorphic as $\mathcal{G}$-fields over $k$ then their fixed subfields $K^{\mathcal{G}}$ and $F^{\mathcal{G}}$ are stably isomorphic over $k^{\mathcal{G}}$.

1.4. Multiplicative $\mathcal{G}$-fields. We are particularly interested in $\mathcal{G}$-fields arising from $\mathcal{G}$-lattices $M$ by first extending the $\mathcal{G}$-action on $M$ to the group algebra $k[M]$ of $M$ over $k$, with $\mathcal{G}$ acting trivially on $k$, and then further to the field of fractions $k(M)=Q(k[M])$. This particular type of $\mathcal{G}$-field will be called multiplicative.

More generally, the above construction can be carried out for any $\mathcal{G}$-field $K$, since the actions of $\mathcal{G}$ on $K$ and $M$ extend uniquely to the group algebra $K[M]$, and then to $K(M)=Q(K[M])$. If the $\mathcal{G}$-action on $K$ is faithful then the field of invariants $K(M)^{\mathcal{G}}$ of the $\mathcal{G}$-field $K(M)$ thus obtained is called the field of tori-invariants of $K$ and $M$ under $\mathcal{G}$.

Various versions of the following proposition can be found in the literature; our particular formulation was motivated by [2, Lemma 2.1].

Proposition. Let $M$ and $N$ be faithful $\mathcal{G}$-lattices. Then the multiplicative $\mathcal{G}$-fields $k(M)$ and $k(N)$ are stably isomorphic over $k$ if and only if $M \sim N$. In particular, the fixed fields $k(M)^{\mathcal{G}}$ and $k(N)^{\mathcal{G}}$ are stably isomorphic over $k$ in this case.

Proof. First, $M \sim N$ implies that $k(M)$ and $k(N)$ are stably isomorphic as $\mathcal{G}$-fields over $k$. Indeed, given the exact sequences (1), the usual "Galois descent" argument (e.g., [17, Prop. 1.3]) shows that $k(E)$ is generated over $k(M)$ by algebraically independent $G$-invariant elements, and similarly for $k(E) / k(N)$.

Conversely, if $k(M)$ and $k(N)$ are stably isomorphic as $\mathcal{G}$-fields over $k$ then, for any $\mathcal{G}$-field $K$ containing $k, K(M)$ and $K(N)$ are stably isomorphic as $\mathcal{G}$-fields over $K$. Indeed, for suitable $r$ and $s$, the fields $k\left(M \oplus \mathbb{Z}^{r}\right)$ and $k\left(N \oplus \mathbb{Z}^{s}\right)$ are isomorphic as $\mathcal{G}$-fields over $k$, and hence $K\left(M \oplus \mathbb{Z}^{r}\right)=Q\left(K \otimes_{k} k\left(M \oplus \mathbb{Z}^{r}\right)\right)$ and $K\left(N \oplus \mathbb{Z}^{s}\right)=$ $Q\left(K \otimes_{k} k\left(N \oplus \mathbb{Z}^{s}\right)\right)$ are isomorphic as $\mathcal{G}$-fields over $K$. As noted in 1.3, this implies that the invariant fields $K(M)^{\mathcal{G}}$ and $K(N)^{\mathcal{G}}$ are stably isomorphic over $K^{\mathcal{G}}$. In particular, choosing $K$ so that $\mathcal{G}$ acts faithfully on $K$, we deduce from [6] (or [21, Theorem 10.1]) that $M \sim N$.

Since the statement about fixed fields has already been explained in 1.3 , the proof is complete. 


\section{Review of the Formanek Strategy}

2.1. Some $\mathcal{S}_{n}$-lattices. We introduce some lattices that will play an important role throughout this note. First, $U_{n}$ denotes the standard permutation $\mathcal{S}_{n}$-lattice of rank $n$; so $U_{n}=\mathbb{Z}_{\uparrow_{\mathcal{S}_{n-1}}^{\mathcal{S}_{n}}}$ or, explicitly,

$$
U_{n}=\mathbb{Z} u_{1} \oplus \ldots \oplus \mathbb{Z} u_{n} \quad \text { with } \mathcal{S}_{n} \text {-action given by } \sigma u_{i}=u_{\sigma(i)} .
$$

Sending each $u_{i} \mapsto 1 \in \mathbb{Z}$, the trivial $\mathcal{S}_{n}$-lattice, yields a map of $\mathcal{S}_{n}$-lattices $\varepsilon: U_{n} \rightarrow \mathbb{Z}$, called the augmentation map. Its kernel is the root lattice $A_{n-1}$. Thus,

$$
A_{n-1}=\left\{\sum_{i=1}^{n} z_{i} u_{i} \mid \sum_{i} z_{i}=0\right\}=\mathbb{Z} a_{1} \oplus \ldots \oplus \mathbb{Z} a_{n-1} \quad \text { with } a_{i}=u_{i}-u_{n} .
$$

Finally, we put

$$
A_{n-1}^{\otimes 2}=A_{n-1} \otimes A_{n-1} .
$$

Tensoring the exact sequence $0 \rightarrow A_{n-1} \longrightarrow U_{n} \stackrel{\varepsilon}{\longrightarrow} \mathbb{Z} \rightarrow 0$ with $A_{n-1}$ we obtain an exact sequence of $\mathcal{S}_{n}$-lattices

$$
0 \rightarrow A_{n-1}^{\otimes 2} \longrightarrow V_{n}=U_{n} \otimes A_{n-1} \longrightarrow A_{n-1} \rightarrow 0 .
$$

Note that the restriction of $A_{n-1}$ to $\mathcal{S}_{n-1}=\operatorname{stab}_{\mathcal{S}_{n}}(n)$ is isomorphic to $U_{n-1}=\mathbb{Z} \uparrow_{\mathcal{S}_{n-2}}^{\mathcal{S}_{n-1}}$. Hence, $V_{n}=\mathbb{Z}_{\mathcal{S}_{n-1}}^{\mathcal{S}_{n}} \otimes A_{n-1} \cong \mathbb{Z} \uparrow_{\mathcal{S}_{n-2}}^{\mathcal{S}_{n}}$.

2.2. The Formanek-Procesi Theorem. Let $M_{n}(k)$ denote the space of $n \times n$ matrices over the field $k$. The group $\mathrm{PGL}_{n}(k)$ operates on $M_{n}(k) \oplus M_{n}(k)$ by simultaneous conjugation. The invariant field of this action is commonly denoted by $C_{n}$; so

$$
C_{n}=k\left(M_{n}(k) \oplus M_{n}(k)\right)^{\mathrm{PGL}_{n}(k)} .
$$

The ultimate goal is to prove (stable) rationality of $C_{n}$ over $k$. Recall that a field extension $F / k$ is called rational if it is purely transcendental and stably rational if there is an extension field $E \supseteq F$ such that $E / F$ and $E / k$ are both rational. Equivalently, $F$ is stably rational over $k$ iff $F$ is stably isomorphic to $k$ over $k$.

The Formanek-Procesi Theorem expresses $C_{n}$ as the multiplicative invariant field arising from the $\mathcal{S}_{n}$-action on the lattice $U_{n} \oplus U_{n} \oplus A_{n-1}^{\otimes 2}$.

Theorem. $C_{n} \cong k\left(U_{n} \oplus U_{n} \oplus A_{n-1}^{\otimes 2}\right)^{\mathcal{S}_{n}}$

This is proved in [10, Theorem 3] building on Procesi's work [19].

2.3. The strategy. Formanek's strategy to prove stable rationality of $C_{n}$ over $k$ consists of finding a faithful $\mathcal{S}_{n}$-lattice $H_{n}$ satisfying

(i) $H_{n} \sim A_{n-1}^{\otimes 2}$, and

(ii) $k\left(H_{n}\right)^{\mathcal{S}_{n}}$ is stably rational over $k$. 
The above formulation of the strategy is taken from [3]. The fact that (i) and (ii) together imply stable rationality of $C_{n}$ over $k$ is immediate from Theorem 2.2 and Proposition 1.4, because $U_{n} \sim 0$ and so $U_{n} \oplus U_{n} \oplus A_{n-1}^{\otimes 2} \sim A_{n-1}^{\otimes 2}$, by Lemma 1.2(i).

\section{The Augmentation Kernel}

3.1. Overview. In this section, we will consider the following situation:

$$
\begin{array}{ll}
U=\mathbb{Z}[X] & \text { will denote a permutation } \mathcal{G} \text {-lattice, } \\
\varepsilon: U \rightarrow \mathbb{Z} & \text { is the augmentation sending all } x \in X \text { to } 1 \text {, and } \\
A=\operatorname{Ker} \varepsilon & \text { will be the augmentation kernel. }
\end{array}
$$

From the exact sequence

$$
1 \rightarrow A \longrightarrow U \stackrel{\varepsilon}{\longrightarrow} \mathbb{Z} \rightarrow 1
$$

one sees that $A$ is quasi-permutation. It is easy to see that $A$ is coflasque or, equivalently, stably permutation precisely if the sizes of the $\mathcal{G}$-orbits in $X$ are coprime. Indeed, from the cohomology sequence that is associated with (3) one obtains $H^{1}(\mathcal{G}, A) \cong \mathbb{Z} / \sum_{\mathcal{O}}|\mathcal{O}| \mathbb{Z}$, where $\mathcal{O}$ runs over the orbits of $\mathcal{G}$ in $X$; cf. Lemma 4.3 below. Thus, $H^{1}(\mathcal{G}, A)=0$ iff the orbit sizes are coprime. On the other hand, the vanishing of $H^{1}(\mathcal{G}, A)=\operatorname{Ext}_{\mathbb{Z}[\mathcal{G}]}(\mathbb{Z}, A)$ forces the splitting of (3)); so $A$ is stably permutation in this case.

By contrast, we will show that the second symmetric power Sym ${ }^{2} A$ of $A$ is always coflasque, and is stably permutation if there is a $\mathcal{G}$-orbit of odd length in $X$. As a consequence, the second exterior power $\bigwedge^{2} A$ of $A$ satisfies

$$
\bigwedge^{2} A \sim A^{\otimes 2}=A \otimes A
$$

in this case. These facts will be proved in (3.4) below after recalling the requisite background concerning Sym ${ }^{2} A$ and $\bigwedge^{2} A$ in (3.2).

3.2. The second symmetric and exterior powers. Let $M$ be a $\mathcal{G}$-lattice. By definition, Sym ${ }^{2} M$ is the quotient of $M^{\otimes 2}=M \otimes M$ modulo the subgroup that is generated by the elements $m \otimes m^{\prime}-m^{\prime} \otimes m$ for $m, m^{\prime} \in M$. We will write $m m^{\prime} \in \operatorname{Sym}^{2} M$ for the image of $m \otimes m^{\prime}$; so $m m^{\prime}=m^{\prime} m$. A $\mathbb{Z}$-basis of $\operatorname{Sym}^{2} M$ is given by $\left\{m_{i} m_{j} \mid 1 \leq i \leq j \leq r\right\}$, where $\left\{m_{1}, \ldots, m_{r}\right\}$ is any $\mathbb{Z}$-basis of $M$. Thus,

$$
\operatorname{rank} \operatorname{Sym}^{2} M=\left(\begin{array}{c}
r+1 \\
2
\end{array}\right) \text { with } r=\operatorname{rank} M \text {. }
$$

Similarly, $\wedge^{2} M$ is the quotient of $M^{\otimes 2}$ modulo the subgroup that is generated by the elements $m \otimes m$ for $m \in M$. Denoting the image of $m \otimes m^{\prime}$ in $\wedge^{2} M$ by $m \wedge m^{\prime}$, a $\mathbb{Z}$-basis of $\bigwedge^{2} M$ is given by $\left\{m_{i} \wedge m_{j} \mid 1 \leq i<j \leq r\right\}$; so

$$
\operatorname{rank} \bigwedge^{2} M=\left(\begin{array}{l}
r \\
2
\end{array}\right) \text {. }
$$


The action of $\mathcal{G}$ on $M^{\otimes 2}$ passes down to $\operatorname{Sym}^{2} M$ and to $\bigwedge^{2} M$, making both $\mathcal{G}$-lattices. The $\mathcal{G}$-lattice $\bigwedge^{2} M$ can be identified with the sublattice of antisymmetric tensors in $M^{\otimes 2}$, that is,

$$
\bigwedge^{2} M \cong \mathrm{A}_{2}^{\prime}(M)=\left\{x \in M^{\otimes 2} \mid x^{\tau}=-x\right\}
$$

where $\tau: M^{\otimes 2} \rightarrow M^{\otimes 2}$ is the switch $\left(m \otimes m^{\prime}\right)^{\tau}=m^{\prime} \otimes m$; see 4 , Exercise 8 on p. A III.190]. Furthermore, $\mathrm{A}_{2}^{\prime}(M)$ is exactly the kernel of the canonical map $M^{\otimes 2} \rightarrow \operatorname{Sym}^{2} M$, and hence we have an exact sequence of $\mathcal{G}$-lattices

$$
0 \rightarrow \bigwedge^{2} M \longrightarrow M^{\otimes 2} \longrightarrow \text { Sym }^{2} M \rightarrow 0
$$

The foregoing, and much of the subsequent material, extends mutatis mutandis to higher tensor, symmetric, and exterior powers of permutation modules, but we will concentrate on squares.

3.3. The case of permutation lattices and their augmentation kernels. Recall that, for a $\mathcal{G}$-set $X$, the cartesian product $X \times X$ becomes a $\mathcal{G}$-set with the diagonal $\mathcal{G}$-action, and the set of 2 -subsets of $X$,

$$
\mathcal{P}_{2}(X)=\{Y \subset X|| Y \mid=2\},
$$

is canonically a $\mathcal{G}$-set as well.

A $\mathcal{G}$-lattice $M$ is called monomial if $M$ has a $\mathbb{Z}$-basis that is permuted by $\mathcal{G}$ up to \pm -sign. Equivalently, $M$ is monomial iff $M$ is a direct sum of lattices that are induced from rank-1 lattices for suitable subgroups $\mathcal{H} \leq \mathcal{G}$. Any $\mathcal{H}$-lattice of rank 1 is given by a homorphism $\varphi: \mathcal{H} \rightarrow\{ \pm 1\}=\mathrm{GL}_{1}(\mathbb{Z})$; we will use the notation $\mathbb{Z}_{\varphi}$ to denote such a lattice. Note that we have the following inclusions:

\{permutation lattices $\} \subset\{$ monomial lattices $\} \subset\{$ quasi-permutation lattices $\}$.

All these inclusions are strict; see (4.4) below.

Lemma. Let $U=\mathbb{Z}[X]$ be a permutation $\mathcal{G}$-lattice and $A$ its augmentation kernel, as in (3.1). Then:

(i) $U^{\otimes 2} \cong \mathbb{Z}[X \times X]$ and $\operatorname{Sym}^{2} U \cong U \oplus \mathbb{Z}\left[\mathcal{P}_{2}(X)\right]$; all these are permutation $\mathcal{G}$ lattices. Furthermore, $\bigwedge^{2} U \cong \oplus_{Y} \mathbb{Z}_{\varphi_{Y}} \uparrow_{\mathcal{G}_{Y}}$ is a monomial lattice. Here, $Y$ runs over a representative set of the $\mathcal{G}$-orbits in $\mathcal{P}_{2}(X), \mathcal{G}_{Y}=\operatorname{stab}_{\mathcal{G}}(Y)$, and $\varphi_{Y}: \mathcal{G}_{Y} \rightarrow\{ \pm 1\}$ is the sign character for the action of $\mathcal{G}_{Y}$ on $Y$.

(ii) There is a $\mathcal{G}$-equivariant exact sequence

$$
0 \rightarrow \operatorname{Sym}^{2} A \rightarrow \mathbb{Z}\left[\mathcal{P}_{2}(X)\right] \rightarrow A / 2 A \rightarrow 0 .
$$

(iii) There is an exact sequence of $\mathcal{G}$-lattices

$$
0 \rightarrow \bigwedge^{2} A \rightarrow \bigwedge^{2} U \rightarrow A \rightarrow 0
$$


Proof. (i) The assertion about $U^{\otimes 2}$ is clear. If $\leq$ is a fixed ordering of $X$ then $\operatorname{Sym}^{2} U$ has $\mathbb{Z}$-basis

$$
\left\{x x^{\prime} \mid x \leq x^{\prime}\right\}=\left\{x^{2} \mid x \in X\right\} \uplus\left\{x x^{\prime} \mid x<x^{\prime}\right\} \cong X \uplus \mathcal{P}_{2}(X)
$$

as $\mathcal{G}$-sets. Thus,

$$
\operatorname{Sym}^{2} U=U^{\prime} \oplus W \quad \text { with } U^{\prime}=\sum_{x} \mathbb{Z} x^{2} \cong U \text { and } W=\sum_{x<x^{\prime}} \mathbb{Z} x x^{\prime} \cong \mathbb{Z}\left[\mathcal{P}_{2}(X)\right] .
$$

Also, $\wedge^{2} U$ has $\mathbb{Z}$-basis $\left\{x \wedge x^{\prime} \mid x<x^{\prime}\right\}$ and $\mathcal{G}$ permutes this basis up to \pm . The description of $\bigwedge^{2} U$ follows.

(ii) The $\mathcal{G}$-map $X \times X \rightarrow U,\left(x, x^{\prime}\right) \mapsto x+x^{\prime}$, gives rise to a map of $\mathcal{G}$-lattices $U^{\otimes 2} \cong \mathbb{Z}[X \times X] \rightarrow U$ which passes down to a map of $\mathcal{G}$-lattices

$$
\rho: \operatorname{Sym}^{2} U \longrightarrow U, \quad x x^{\prime} \mapsto x+x^{\prime} \quad\left(x, x^{\prime} \in X\right) .
$$

One easily checks that $\operatorname{Im} \rho=\varepsilon^{-1}(2 \mathbb{Z})$. Since $\operatorname{Sym}^{2} A \subset \operatorname{Sym}^{2} U$ is spanned by the elements of the form $\left(x-x^{\prime}\right)\left(y-y^{\prime}\right)$ for $x, x^{\prime}, y, y^{\prime} \in X$, and

$$
\rho\left(\left(x-x^{\prime}\right)\left(y-y^{\prime}\right)\right)=x+y-\left(x^{\prime}+y\right)-\left(x+y^{\prime}\right)+x^{\prime}+y^{\prime}=0,
$$

we have $\operatorname{Sym}^{2} A \subset \operatorname{Ker} \rho$. Now, $\operatorname{Sym}^{2} A$ is a pure sublattice of $\operatorname{Sym}^{2} U$ and

$$
\operatorname{rank} \operatorname{Sym}^{2} U-\operatorname{rank} \operatorname{Sym}^{2} A=\left(\begin{array}{c}
|X|+1 \\
2
\end{array}\right)-\left(\begin{array}{c}
|X| \\
2
\end{array}\right)=|X|=\operatorname{rank} \operatorname{Im} \rho .
$$

This implies that, in fact, $\operatorname{Sym}^{2} A=\operatorname{Ker} \rho$. Thus, we obtain an exact sequence of $\mathcal{G}$-lattices

$$
0 \rightarrow \text { Sym }^{2} A \stackrel{\text { incl. }}{\longrightarrow} \text { Sym }^{2} U \stackrel{\rho}{\longrightarrow} \varepsilon^{-1}(2 \mathbb{Z}) \rightarrow 0 \text {. }
$$

Using the above decomposition $\operatorname{Sym}^{2} U=U^{\prime} \oplus W$ and noting that $\rho\left(U^{\prime}\right)=2 U$, we deduce the following exact sequence

$$
0 \rightarrow U^{\prime}+\text { Sym }^{2} A \stackrel{\text { incl. }}{\longrightarrow} \text { Sym }^{2} U=U^{\prime} \oplus W \stackrel{\bar{\rho}}{\longrightarrow} \varepsilon^{-1}(2 \mathbb{Z}) / 2 U \rightarrow 0 .
$$

The sum $U^{\prime}+\operatorname{Sym}^{2} A \subset \operatorname{Sym}^{2} U$ is direct, as follows by counting ranks. Furthermore, using the notation $-=(.) \otimes \mathbb{Z} / 2 \mathbb{Z}$ for reduction $\bmod 2$, we have $\varepsilon^{-1}(2 \mathbb{Z}) / 2 U=$ $\operatorname{Ker}(\bar{U} \stackrel{\bar{\varepsilon}}{\rightarrow} \overline{\mathbb{Z}})=\bar{A}$. Therefore, we obtain an exact sequence of $\mathcal{G}$-modules

$$
0 \rightarrow \operatorname{Sym}^{2} A \longrightarrow W \stackrel{\bar{\rho}}{\longrightarrow} \bar{A}=A / 2 A \rightarrow 0
$$

proving part (ii).

(iii) Consider the map (a piece of the Koszul complex)

$$
\pi: \bigwedge^{2} U \longrightarrow A, \quad x \wedge x^{\prime} \mapsto x-x^{\prime} \quad\left(x<x^{\prime}\right) .
$$

This map is $\mathcal{G}$-equivariant and surjective, and one checks that $\bigwedge^{2} A \subset \bigwedge^{2} U$ is contained in the kernel. Counting ranks, one sees that, in fact, $\bigwedge^{2} A=\operatorname{Ker} \pi$, which completes the proof. 
The sequence in part (iii) above, for $\bigwedge^{2} A$, plays a role analogous to that of sequence (2) for $A_{n-1}^{\otimes 2}$.

3.4. Sym $^{2} A$ is stably permutation. We are now ready to prove the results announced in (3.1).

Proposition. The $\mathcal{G}$-lattice $\mathrm{Sym}^{2} A$ is coflasque. If there is a $\mathcal{G}$-orbit of odd length in $X$ then $\mathrm{Sym}^{2} A$ is stably permutation. Consequently, $\bigwedge^{2} A \sim A^{\otimes 2}$ holds in this case.

Proof. Continuing with the notation used in the proof of Lemma 3.3, we first show that $\mathrm{Sym}^{2} A$ is coflasque. Inasmuch as the permutation $\mathcal{G}$-lattice $W$ is certainly coflasque, the sequence (5) reduces the assertion to the following statement about fixed points:

$$
W^{\mathcal{H}} \stackrel{\bar{\rho}}{\longrightarrow} \bar{A}^{\mathcal{H}} \quad \text { is epi for all } \mathcal{H} \leq \mathcal{G} .
$$

Now $\bar{A}^{\mathcal{H}}=\bar{U}^{\mathcal{H}} \cap \operatorname{Ker}(\bar{U} \stackrel{\bar{\varepsilon}}{\rightarrow} \overline{\mathbb{Z}})$, and $\bar{U}^{\mathcal{H}}$ has $\overline{\mathbb{Z}}$-basis the orbit sums $\widehat{\mathcal{O}}=\sum_{x \in \mathcal{O}} \bar{x}$, where $\mathcal{O}$ is an $\mathcal{H}$-orbit in $X$. Therefore, $\bar{A}^{\mathcal{H}}$ is spanned by the orbit sums $\widehat{\mathcal{O}}$, where $\mathcal{O}$ has even length, together with the elements $\widehat{\mathcal{O}}+\widehat{\mathcal{O}^{\prime}}$ with $\mathcal{O}$ and $\mathcal{O}^{\prime}$ both of odd length. Both types can be written as $\widehat{E}=\sum_{x \in E} \bar{x}$, where $E \subset X$ is an $\mathcal{H}$-invariant subset of even size. For any $\mathcal{H}$-invariant $E \subset X$, say $E=\left\{x_{1}, \ldots, x_{e}\right\}$, put $w_{E}=$ $\sum_{1 \leq i<j \leq e} x_{i} x_{j}$, an $\mathcal{H}$-invariant element of $W$ (note that $x_{i} x_{j}=x_{j} x_{i}$ ). The image $\bar{\rho}\left(w_{E}\right) \in \bar{A}^{\mathcal{H}}$ is

$$
\sum_{1 \leq i<j \leq e} \overline{x_{i}}+\overline{x_{j}}=(e-1) \sum_{i=1}^{e} \overline{x_{i}}= \begin{cases}\overline{x_{1}}+\ldots+\overline{x_{e}} & \text { if } e \text { is even; } \\ 0 & \text { if } e \text { is odd }\end{cases}
$$

Thus, if $e$ is even then $\bar{\rho}\left(w_{E}\right)=\widehat{E}$, and so $(\sqrt{6})$ is proved.

If there is an odd length $\mathcal{G}$-orbit $\mathcal{O} \subset X$ then $\bar{U}=\bar{A} \oplus \overline{\mathbb{Z}} \widehat{\mathcal{O}}$. Thus, we can extend the map $\bar{\rho}$ in (5) to a $\mathcal{G}$-equivariant surjection $W \oplus \mathbb{Z} \rightarrow \bar{U}$ by sending $1 \in \mathbb{Z}$ to $\widehat{\mathcal{O}}$. This leads to an exact sequence of $\mathcal{G}$-modules

$$
0 \rightarrow \operatorname{Sym}^{2} A \oplus \mathbb{Z} \longrightarrow W \oplus \mathbb{Z} \longrightarrow \bar{U} \rightarrow 0
$$

Since $U$ and $W \oplus \mathbb{Z}$ are permutation lattices, Lemma 1.1 (with $\alpha=\cdot 2$ ) yields an exact sequence

$$
0 \rightarrow \operatorname{Sym}^{2} A \oplus \mathbb{Z} \longrightarrow W \oplus \mathbb{Z} \oplus U \longrightarrow U \rightarrow 0
$$

Finally, since Sym ${ }^{2} A \oplus \mathbb{Z}$ is coflasque, this sequence splits which proves that $\operatorname{Sym}^{2} A$ is stably permutation.

In view of the sequence (4), the assertion about $\bigwedge^{2} A$ now follows from Lemma 1.2(ii). 
3.5. The root lattice $A_{n-1}$. Taking $\mathcal{G}=\mathcal{S}_{n}$ and $X=\left\{u_{1}, \ldots, u_{n}\right\} \cong \mathcal{S}_{n} / \mathcal{S}_{n-1}$, as in (2.1), we have $U=U_{n}$ and $A=A_{n-1}$. Furthermore, $W=\bigoplus_{i<j} \mathbb{Z} u_{i} u_{j} \cong W_{n}:=$ $\mathbb{Z} \uparrow \mathcal{S}_{\mathcal{S}_{n-2} \times \mathcal{S}_{2}}$ and

$$
\bigwedge^{2} U_{n} \cong \mathbb{Z}_{\varphi} \uparrow \mathcal{S}_{\mathcal{S}_{n-2} \times \mathcal{S}_{2}}
$$

where $\varphi: \mathcal{S}_{n-2} \times \mathcal{S}_{2} \rightarrow\{ \pm 1\}$ is trivial on $\mathcal{S}_{n-2}$. For odd $n$, the split sequence (7) gives the following isomorphism of $\mathcal{S}_{n}$-lattices:

$$
\operatorname{Sym}^{2} A_{n-1} \oplus U_{n} \oplus \mathbb{Z} \cong W_{n} \oplus U_{n} \oplus \mathbb{Z} .
$$

For $n=2$, Sym $^{2} A_{1} \cong \mathbb{Z}$ is the trivial $\mathcal{S}_{2}$-lattice and, for $n=4$, Formanek [11, Lemma $5(1)$ ] has shown that

$$
\operatorname{Sym}^{2} A_{3} \oplus \mathbb{Z} \cong U_{4} \oplus \mathbb{Z}_{\uparrow}^{\mathcal{S}_{\mathcal{D}}}
$$

where $\mathcal{D}$ is the Sylow 2-subgroup of $\mathcal{S}_{4}$.

Proposition 3.4 gives the equivalence

$$
\bigwedge^{2} A_{n-1} \sim A_{n-1}^{\otimes 2}
$$

for odd $n$ and for $n=2,4$. (We will see later (4.5) that (8) fails to hold for even $n \geq 6$.) In view of (2.3), the equivalence (8) reduces the rationality problem for $C_{n}$, for odd $n$, to the corresponding problem for the $\mathcal{S}_{n}$-lattice $\bigwedge^{2} A_{n-1}$ :

$$
\text { Is } k\left(\bigwedge^{2} A_{n-1}\right)^{\mathcal{S}_{n}} \text { (stably) rational over } k \text { ? }
$$

Rationally, $\bigwedge^{2} A_{n-1}$ is irreducible. In fact,

$$
\bigwedge^{2} A_{n-1} \otimes \mathbb{Q} \cong S^{\left(n-2,1^{2}\right)}
$$

where $S^{\left(n-2,1^{2}\right)}$ is the Specht module corresponding to the partition $\left(n-2,1^{2}\right)$ of $n$; e.g., [9, Exercise 4.6 on p. 48].

Here are some small cases:

3.5.1. The case $n=2$. Clearly, $\bigwedge^{2} A_{1}=0$, and so (9) is trivial.

3.5.2. The case $n=3$. Since $\bigwedge^{2} A_{2}=\mathbb{Z} \cdot a_{1} \wedge a_{2}$, with $a_{i}$ as in (2.1), one sees that

$$
\bigwedge^{2} A_{2} \cong \mathbb{Z}^{-} \sim 0
$$

So, $k\left(\bigwedge^{2} A_{2}\right)=k(t)$, with $(1,2) \cdot t=t^{-1}$ and with $(1,2,3)$ acting trivially. The invariant field is $k\left(t+t^{-1}\right)$, a rational extension of $k$. (This is also clear from Lüroth's Theorem.) 
3.5.3. The case $n=4$. Here,

$$
\bigwedge^{2} A_{3} \cong \operatorname{Hom}_{\mathbb{Z}}\left(A_{3}, \mathbb{Z}^{-}\right)
$$

This isomorphism is a consequence of the following equalities of $\mathcal{S}_{4}$-lattices:

$$
\left(\bigwedge^{2} A_{3}\right) \wedge A_{3}=\bigwedge^{3} A_{3}=\mathbb{Z}_{\operatorname{det} A_{3}}=\mathbb{Z}^{-}
$$

Further, $\operatorname{Hom}_{\mathbb{Z}}\left(A_{3}, \mathbb{Z}^{-}\right) \cong A_{3}^{*} \otimes \mathbb{Z}^{-} \cong\left(S A_{3}\right)^{*}$, the dual of the signed root lattice $S A_{3}=\mathbb{Z}^{-} \otimes A_{3}$. Formanek has shown [11, Theorems 13 and 14] that $k\left(S A_{3}^{*}\right)^{\mathcal{S}_{4}}$ is rational over $k$. The same result is also covered as case $W_{8}(198)$ in [12].

\section{Picard Groups}

4.1. Statement of results. Following [6, 7] we put, for any $\mathbb{Z}[\mathcal{G}]$-module $M$,

$$
\amalg^{i}(\mathcal{G}, M)=\bigcap_{g \in \mathcal{G}} \operatorname{Ker}\left(\operatorname{Res}_{\langle g\rangle}^{\mathcal{G}}: H^{i}(\mathcal{G}, M) \rightarrow H^{i}(\langle g\rangle, M)\right) .
$$

Of particular interest for us will be the case where $M$ is a $\mathcal{G}$-lattice and $i=1$ or 2 . Indeed, if $\sim$ denotes the equivalence of (1.2) then

$$
\amalg^{2}(\mathcal{G}, .) \text { is constant on } \sim \text {-classes; }
$$

see Lemma 4.2 below. Furthermore, by [18], one has

$$
\amalg^{1}(\mathcal{G}, M)=\operatorname{Pic}\left(k[M]^{\mathcal{G}}\right),
$$

the Picard group of the algebra of invariants under the (multiplicative) action of $\mathcal{G}$ on the group algebra $k[M]$. These Picard groups are seldom nontrivial: If $\operatorname{rank} M=3$ then only 2 out of the 73 conjugacy classes of finite subgroups $\mathcal{G} \leq \mathrm{GL}_{3}(\mathbb{Z})$ lead to invariants with nontrivial Picard group, and only 9 out of 710 occur for rank $M=4$; see [18. Nevertheless, we will prove here the following nontriviality result for the root lattice $M=A_{n-1}$. Note that, since $A_{n-1} \sim 0$, the result shows in particular that $\amalg^{1}(\mathcal{G}$, . ) is not in general a $\sim$-invariant.

Proposition. (i) Suppose $n$ is not prime and let $p$ be the smallest prime divisor of $n$. Then there exists a subgroup $\mathcal{H} \cong C_{p} \times C_{p}$ of $\mathcal{S}_{n}$ such that $\amalg^{1}\left(\mathcal{H}, A_{n-1}\right) \cong$ $\mathbb{Z} / p \mathbb{Z}$.

(ii) If $n=2^{a} \geq 4$ then $\amalg^{1}\left(\mathcal{A}_{n}, A_{n-1}\right) \cong \amalg^{1}\left(\mathcal{T}, A_{n-1}\right) \cong \mathbb{Z} / 2 \mathbb{Z}$, where $\mathcal{A}_{n}$ is the alternating group of degree $n$ and $\mathcal{T}$ denotes the Sylow 2-subgroup of $\mathcal{A}_{n}$.

Part (ii), with $n=4$, yields the two nontrivial Picard groups in rank 3. The following corollary of the proposition confirms a conjecture of Le Bruyn [16, p. 108].

Corollary. If $n$ is not prime then the $\mathcal{S}_{n}$-lattice $A_{n-1}^{\otimes 2}$ is not equivalent to a direct summand of a quasi-permutation lattice. 
This corollary will be immediate from part (i) of the Proposition together with Lemma 4.2 below. Inasmuch as $A_{n-1}^{\otimes 2}$ is known to be permutation projective for prime values of $n$ [3, Proposition 3], our non-primeness hypothesis on $n$ is definitely necessary. We remark that $A_{n-1}^{\otimes 2}$ was previously known not to be quasi-permutation for all $n$ that are divisible by a square [20] or whenever $n$ is a prime $>3$ [3, Corollary 1(a)]. Summarizing, we now know:

The $\mathcal{S}_{n}$-lattice $A_{n-1}^{\otimes 2}$ is quasi-permutation precisely for $n=2$ and $n=3$.

Finally, we consider even values of $n \geq 6$ and show in (4.5) that $\bigwedge^{2} A_{n-1}$ is no longer equivalent to $A_{n-1}^{\otimes 2}$ for such $n$.

4.2. Connection with equivalence of lattices. Every $\mathcal{G}$-lattice $M$ has a flasque resolution

$$
0 \rightarrow M \rightarrow P \rightarrow F \rightarrow 0
$$

with $P$ a permutation $\mathcal{G}$-lattice and $F$ a flasque $\mathcal{G}$-lattice, that is, $H^{-1}(\mathcal{H}, F)=0$ holds for all subgroups $\mathcal{H} \leq \mathcal{G}$. Moreover, $F$ is determined by $M$ up to stable equivalence: If $0 \rightarrow M \rightarrow P^{\prime} \rightarrow F^{\prime} \rightarrow 0$ is another flasque resolution of $M$ then $F$ and $F^{\prime}$ are stably equivalent, that is, $F \oplus Q \cong F^{\prime} \oplus Q^{\prime}$ holds for suitable permutation $\mathcal{G}$-lattices $Q$ and $Q^{\prime}$. Following [6], the stable equivalence class of $M$ will be written $[M]$, and the stable equivalence class of $F$ in the flasque resolution (10) will be denoted $\rho(M) ;$ so

$$
\rho(M)=[F] .
$$

Finally, for any two $\mathcal{G}$-lattices $M$ and $N$,

$$
M \sim N \Longleftrightarrow \rho(M)=\rho(N) \text {. }
$$

See [6, Lemme 5] for all this.

Note that $H^{ \pm 1}(\mathcal{G}, M)$ depends only on the stable equivalence class $[M]$, because $H^{ \pm 1}$ is trivial for permutation modules. The following lemma is extracted from [7, pp. 199-202].

Lemma. (i) Let $0 \rightarrow M \rightarrow P \rightarrow N \rightarrow 0$ be an exact sequence of $\mathbb{Z}[\mathcal{G}]$-modules, with $P$ a permutation projective $\mathcal{G}$-lattice. Then $\amalg^{2}(\mathcal{G}, M) \cong \amalg^{1}(\mathcal{G}, N)$.

(ii) For any $\mathcal{G}$-lattice $M, H^{1}(\mathcal{G}, \rho(M)) \cong \amalg^{2}(\mathcal{G}, M)$.

(iii) If $M$ is equivalent to a direct summand of a quasi-permutation $\mathcal{G}$-lattice then $\amalg^{2}(\mathcal{H}, M)=0$ holds for all subgroups $\mathcal{H} \leq \mathcal{G}$.

Proof. (i) First, $\amalg^{2}(\mathcal{G}, P)=0$, because $\amalg^{i}(\mathcal{G},$.$) is additive on direct sums and$ $H^{2}(\mathcal{G}, \mathbb{Z} \uparrow \mathcal{G}) \cong \operatorname{Hom}(\mathcal{H}, \mathbb{Q} / \mathbb{Z})$ is detected by restrictions to cyclic subgroups. The isomorphism $\amalg^{2}(\mathcal{G}, M) \cong \amalg^{1}(\mathcal{G}, N)$ now follows from the commutative exact diagram below, obtained from the cohomology sequences that are associated with the given 
exact sequence:

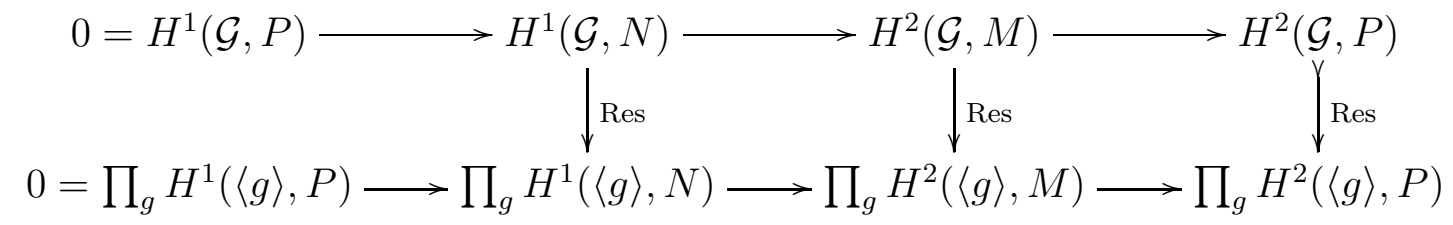

(ii) Let $0 \rightarrow M \rightarrow P \rightarrow F \rightarrow 0$ be a flasque resolution of $M$; so $\rho(M)=[F]$. By periodicity of cohomology for cyclic groups, we have $H^{1}(\langle g\rangle, \rho(M)) \cong H^{-1}(\langle g\rangle, \rho(M))=$ 0 for all $g \in \mathcal{G}$, because $F$ is flasque. Thus, $\amalg^{1}(\mathcal{G}, \rho(M))=H^{1}(\mathcal{G}, \rho(M))$ and the isomorphism $H^{1}(\mathcal{G}, \rho(M)) \cong \amalg^{2}(\mathcal{G}, M)$ follows from part (i).

(iii) Since the property of being equivalent to a direct summand of a quasi-permutation lattice is inherited by restrictions to subgroups, it suffices to show that $\amalg^{2}(\mathcal{G}, M)=0$ or, equivalently, $H^{1}(\mathcal{G}, \rho(M))=0$ holds whenever $M \oplus M^{\prime}$ is quasi-permutation. But $\rho($.$) is easily seen to be additive, as is H^{1}(\mathcal{G}$, . $)$. So $H^{1}(\mathcal{G}, \rho(M)) \oplus H^{1}\left(\mathcal{G}, \rho\left(M^{\prime}\right)\right) \cong$ $H^{1}\left(\mathcal{G}, \rho\left(M \oplus M^{\prime}\right)\right)=0$, because $\rho\left(M \oplus M^{\prime}\right)=[0]$. This entails that $H^{1}(\mathcal{G}, \rho(M))=0$, as desired.

Deduction of Corollary 4.1 from Proposition 4.1 (i). Applying part (i) of the Lemma to the sequence (2), we deduce the isomorphism

$$
\amalg^{2}\left(\mathcal{H}, A_{n-1}^{\otimes 2}\right) \cong \amalg^{1}\left(\mathcal{H}, A_{n-1}\right)
$$

for any subgroup $\mathcal{H} \leq \mathcal{S}_{n}$. Inasmuch as $\amalg^{1}\left(\mathcal{H}, A_{n-1}\right)$ is nontrivial for certain $\mathcal{H}$ if $n$ is not prime, by Proposition 4.1(i), we conclude from part (iii) of the Lemma that the $\mathcal{S}_{n}$-lattice $A_{n-1}^{\otimes 2}$ is not equivalent to a direct summand of a quasi-permutation lattice, as asserted in Corollary 4.1.

4.3. Proof of Proposition 4.1. We begin with a simple lemma which shows in particular that the restriction map $H^{1}\left(\mathcal{S}_{n}, A_{n-1}\right) \rightarrow H^{1}\left(\langle\tau\rangle, A_{n-1}\right)$ is an isomorphism for $\tau=(1,2, \ldots, n)$; so $\amalg^{1}\left(\mathcal{S}_{n}, A_{n-1}\right)=\{0\}$.

Lemma. For any subgroup $\mathcal{H} \leq \mathcal{S}_{n}, H^{1}\left(\mathcal{H}, A_{n-1}\right) \cong \mathbb{Z} / \sum_{\mathcal{O}}|\mathcal{O}| \mathbb{Z}$, where $\mathcal{O}$ runs over the orbits of $\mathcal{H}$ in $\{1, \ldots, n\}$. Moreover, the restriction map $H^{1}\left(\mathcal{S}_{n}, A_{n-1}\right) \rightarrow$ $H^{1}\left(\mathcal{H}, A_{n-1}\right)$ is surjective.

Proof. From the cohomology sequence that is associated with the augmentation sequence $1 \rightarrow A_{n-1} \rightarrow U_{n}=\mathbb{Z}[\{1, \ldots, n\}] \stackrel{\varepsilon}{\rightarrow} \mathbb{Z} \rightarrow 1$, one obtains the exact sequence $U_{n}^{\mathcal{H}} \stackrel{\varepsilon}{\rightarrow} \mathbb{Z} \rightarrow H^{1}\left(\mathcal{H}, A_{n-1}\right) \rightarrow 0$ which implies the asserted description of $H^{1}\left(\mathcal{H}, A_{n-1}\right)$. For surjectivity of restriction maps, consider the commutative diagram of cohomology sequences

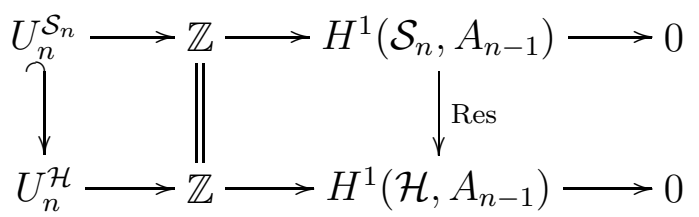


Proof of Proposition 4.1 (i). Let $p$ be the smallest prime divisor of $n$; so $n=p k$ with $k \geq p$. Define commuting $p$-cycles $\sigma_{1}, \ldots, \sigma_{k} \in \mathcal{S}_{n}$ by

$$
\sigma_{i}=((i-1) p+1,(i-1) p+2, \ldots, i p) .
$$

First assume $k \geq p+1$. Define $\alpha, \beta \in \mathcal{S}_{n}$ by

$$
\alpha=\prod_{i=1}^{k-1} \sigma_{i} \quad \text { and } \quad \beta=\prod_{i=1}^{p-1} \sigma_{i}^{-i} \cdot \prod_{i=p+1}^{k} \sigma_{i} .
$$

Put $\mathcal{H}=\langle\alpha, \beta\rangle \leq \mathcal{S}_{n}$, an elementary abelian $p$-group of rank 2 . The $\mathcal{H}$-orbits in $\{1,2, \ldots, n\}$ are exactly the subsets $\mathcal{O}_{i}=\{(i-1) p+1,(i-1) p+2, \ldots, i p\}$, all of length $p$. Therefore, by the Lemma,

$$
H^{1}\left(\mathcal{H}, A_{n-1}\right) \cong \mathbb{Z} / p \mathbb{Z} .
$$

The cyclic subgroups $\mathcal{C} \leq \mathcal{H}$ are: $\langle 1\rangle,\langle\alpha\rangle$ (acts trivially on $\mathcal{O}_{k}$ ), and $\left\langle\alpha^{i} \beta\right\rangle(1 \leq i \leq p)$ (acts trivially on $\left.\mathcal{O}_{i}\right)$. Since they all have fixed points, $H^{1}\left(\mathcal{C}, A_{n-1}\right)$ is trivial in each case. Therefore, $\amalg^{1}\left(\mathcal{H}, A_{n-1}\right)=H^{1}\left(\mathcal{H}, A_{n-1}\right)=\mathbb{Z} / p \mathbb{Z}$, as required.

Now assume $n=p^{2}$ and define commuting $p$-cycles $\pi_{1}, \ldots, \pi_{p} \in \mathcal{S}_{n}$ by

$$
\pi_{i}=(i, i+p, \ldots, i+p(p-1)) .
$$

(Imagining the numbers $1, \ldots, p^{2}$ arranged in a square with $i$-th row the entries of $\sigma_{i}$, the $\pi_{i}$ are defined columnwise.) Put $\pi=\prod_{i=1}^{p} \pi_{i}$. Then $\pi \sigma_{i} \pi^{-1}=\sigma_{i+1}$ (indices $\bmod p$ ), and so $\pi$ commutes with $\sigma=\prod_{i=1}^{p} \sigma_{i}$. Put $\mathcal{H}=\langle\sigma, \pi\rangle \leq \mathcal{S}_{n}$, so $\mathcal{H} \cong C_{p} \times C_{p}$. Note that $\mathcal{H}$ acts transitively on $\left\{1, \ldots, p^{2}\right\}$, and hence $\left\{1, \ldots, p^{2}\right\} \cong \mathcal{H}$ as left $\mathcal{H}$ sets. Therefore, the orbits of every nonidentity element of $\mathcal{H}$ all have length $p$. The Lemma now gives

$$
H^{1}\left(\mathcal{H}, A_{n-1}\right) \cong \mathbb{Z} / p^{2} \mathbb{Z} \quad \text { and } \quad H^{1}\left(\langle g\rangle, A_{n-1}\right) \cong \mathbb{Z} / p \mathbb{Z}
$$

for each nonidentity $g \in \mathcal{H}$. Since the restriction maps $H^{1}\left(\mathcal{H}, A_{n-1}\right) \rightarrow H^{1}\left(\langle g\rangle, A_{n-1}\right)$ are surjective, by the Lemma, the kernel is the socle of $\mathbb{Z} / p^{2} \mathbb{Z}$ in each case. This socle is therefore equal to $\amalg^{1}\left(\mathcal{H}, A_{n-1}\right)$, which completes the proof of part (i).

Proof of Proposition 4.1(ii). Now assume $n=2^{a} \geq 4$. The Lemma implies that $H^{1}\left(\mathcal{A}_{n}, A_{n-1}\right) \cong \mathbb{Z} / n \mathbb{Z}$ is a 2 -group. Thus, the restriction map $H^{1}\left(\mathcal{A}_{n}, A_{n-1}\right) \rightarrow$ $H^{1}\left(\mathcal{T}, A_{n-1}\right)$ is an isomorphism for the Sylow 2-subgroup $\mathcal{T}$ of $\mathcal{A}_{n}$; cf. [5], p. 84]. Therefore, $\amalg^{1}\left(\mathcal{A}_{n}, A_{n-1}\right)$ embeds into $\amalg^{1}\left(\mathcal{T}, A_{n-1}\right)$. We may assume that $\tau=$ $(1,2, \ldots, n)^{2} \in \mathcal{T}$. Since $H^{1}\left(\mathcal{T}, A_{n-1}\right) \cong \mathbb{Z} / n \mathbb{Z}$ maps onto $H^{1}\left(\langle\tau\rangle, A_{n-1}\right) \cong \mathbb{Z} / \frac{n}{2} \mathbb{Z}$, we see that $\amalg^{1}\left(\mathcal{T}, A_{n-1}\right)$ has order at most 2 . On the other hand, since $\mathcal{A}_{n}$ contains no $n$-cycle, all $H^{1}\left(\langle g\rangle, A_{n-1}\right)$ with $g \in \mathcal{A}_{n}$ are cyclic 2 -groups of order smaller than $n$. Hence the socle of $H^{1}\left(\mathcal{A}_{n}, A_{n-1}\right) \cong \mathbb{Z} / n \mathbb{Z}$ maps to 0 under all restrictions $H^{1}\left(\mathcal{A}_{n}, A_{n-1}\right) \rightarrow H^{1}\left(\langle g\rangle, A_{n-1}\right)$, and so the socle is contained in $\amalg^{1}\left(\mathcal{A}_{n}, A_{n-1}\right)$. 
Therefore, $\amalg^{1}\left(\mathcal{A}_{n}, A_{n-1}\right)$ and $\amalg^{1}\left(\mathcal{T}, A_{n-1}\right)$ both have order 2 , and the proof of the Proposition is complete.

4.4. Monomial lattices. This section contains some observations about monomial lattices; see (3.3). In particular, we will show that their $H^{1}$ is always an elementary abelian 2-group. Therefore, the quasi-permutation $\mathcal{S}_{n}$-lattice $A_{n-1}$ is not monomial for $n>2$.

Lemma. Let $M$ be a monomial $\mathcal{G}$-lattice; so $M$ is a direct sum of lattices isomorphic to $\mathbb{Z}_{\varphi} \uparrow_{\mathcal{H}}$ for $\mathcal{H} \leq \mathcal{G}$ and $\varphi: \mathcal{H} \rightarrow\{ \pm 1\}$. Then $H^{1}(\mathcal{G}, M) \cong(\mathbb{Z} / 2 \mathbb{Z})^{m}$, where $m$ is the number of nontrivial $\varphi$ 's occuring in the sum. Furthermore, $\amalg^{1}(\mathcal{G}, M)=\{0\}$ and $\amalg^{2}(\mathcal{G}, M)=\{0\}$.

Proof. Since monomial lattices are quasi-permutation, we know by Lemma 4.2(iii) that $\amalg^{2}(\mathcal{G}, M)=\{0\}$; so we may concentrate on $H^{1}$ and $\Psi^{1}$. As both functors are additive on direct sums, we may assume that $M=\mathbb{Z}_{\varphi} \uparrow \mathcal{G}$ with $\varphi: \mathcal{H} \rightarrow\{ \pm 1\}$ nontrivial (otherwise, $M$ is a permutation module and the assertions are clear). Then

$$
H^{1}(\mathcal{G}, M) \cong H^{1}\left(\mathcal{H}, \mathbb{Z}_{\varphi}\right) \cong H^{1}\left(\mathcal{H} / \operatorname{Ker} \varphi, \mathbb{Z}_{\varphi}\right)=\mathbb{Z} / 2 \mathbb{Z},
$$

where the first isomorphism comes from Shapiro's Lemma and the second is given by inflation; see [1, 34.2 and 35.3]. This proves the asserted isomorphism for $H^{1}(\mathcal{G}, M)$.

The Shapiro isomorphism $H^{1}(\mathcal{G}, M) \stackrel{\cong}{\longrightarrow} H^{1}\left(\mathcal{H}, \mathbb{Z}_{\varphi}\right)$ is equal to the composite

$$
\operatorname{proj}^{*} \circ \operatorname{Res}_{\mathcal{H}}^{\mathcal{G}}: H^{1}(\mathcal{G}, M) \rightarrow H^{1}(\mathcal{H}, M) \rightarrow H^{1}\left(\mathcal{H}, \mathbb{Z}_{\varphi}\right)
$$

where proj : $M=\mathbb{Z}_{\varphi} \uparrow_{\mathcal{H}}^{\mathcal{G}} \rightarrow \mathbb{Z}_{\varphi}$ is the projection onto the direct summand $1 \otimes \mathbb{Z}_{\varphi} \cong \mathbb{Z}_{\varphi}$ of $M$. Fixing $g \in \mathcal{H}$ with $\varphi(g)=-1$, the restriction map $H^{1}\left(\mathcal{H}, \mathbb{Z}_{\varphi}\right)=\mathbb{Z} / 2 \mathbb{Z} \rightarrow$ $H^{1}\left(\langle g\rangle, \mathbb{Z}_{\varphi}\right)=\mathbb{Z} / 2 \mathbb{Z}$ is an isomorphism, and hence so is the map $\operatorname{Res}_{\langle g\rangle}^{\mathcal{H}} \circ \operatorname{proj}^{*} \circ \operatorname{Res}_{\mathcal{H}}^{\mathcal{G}}=$ $\operatorname{proj}^{*} \circ \operatorname{Res}_{\langle g\rangle}^{\mathcal{G}}$. This proves that $\operatorname{Res}_{\langle g\rangle}^{\mathcal{G}}: H^{1}(\mathcal{G}, M) \rightarrow H^{1}(\langle g\rangle, M)$ is injective, whence $\amalg^{1}(\mathcal{G}, M)=\{0\}$.

4.5. The case of even $n \geq 6$. As promised in (3.5), we now use the above techniques to prove the following

Lemma. For even $n \geq 6$, the $\mathcal{S}_{n}$-lattice $\operatorname{Sym}^{2} A_{n-1}$ is not equivalent to a direct summand of a quasi-permutation lattice. Moreover, $\bigwedge^{2} A_{n-1} \nsim A_{n-1}^{\otimes 2}$.

Proof. We first deal with $\mathrm{Sym}^{2} A_{n-1}$. By Lemma 4.2(iii), it suffices to show that $\amalg^{2}\left(\mathcal{H}, \operatorname{Sym}^{2} A_{n-1}\right) \neq 0$ holds for some subgroup $\mathcal{H} \leq \mathcal{S}_{n}$. To this end, we use the exact sequence of Lemma 3.3(ii) which now takes the following form (cf. (3.5))

$$
0 \rightarrow \text { Sym }^{2} A_{n-1} \longrightarrow \mathbb{Z}_{\mathcal{S}_{n-2} \times \mathcal{S}_{2}}^{\mathcal{S}_{n}} \longrightarrow \overline{A_{n-1}}=A_{n-1} / 2 A_{n-1} \rightarrow 0
$$

where $-=(.) \otimes \mathbb{Z} / 2 \mathbb{Z}$ denotes reduction $\bmod 2$. By Lemma 4.2(i), this sequence implies $\amalg^{2}\left(\mathcal{H}, \operatorname{Sym}^{2} A_{n-1}\right) \cong \amalg^{1}\left(\mathcal{H}, \overline{A_{n-1}}\right)$, and hence the issue is to show that $\amalg^{1}\left(\mathcal{H}, \overline{A_{n-1}}\right) \neq 0$ holds for some subgroup $\mathcal{H} \leq \mathcal{S}_{n}$. Now, suppose we can find a subgroup $\mathcal{H}$ satisfying 
1. $\mathcal{H}$ is a 2 -group;

2. $\mathcal{H}$ has no fixed point in $\{1,2, \ldots, n\}$, but every $g \in \mathcal{H}$ does have a fixed point. Then the cohomology sequence that is associated with the exact sequence $0 \rightarrow$ $\overline{A_{n-1}} \rightarrow \overline{U_{n}} \stackrel{\bar{\varepsilon}}{\rightarrow} \overline{\mathbb{Z}} \rightarrow 0$ yields the following commutative diagram

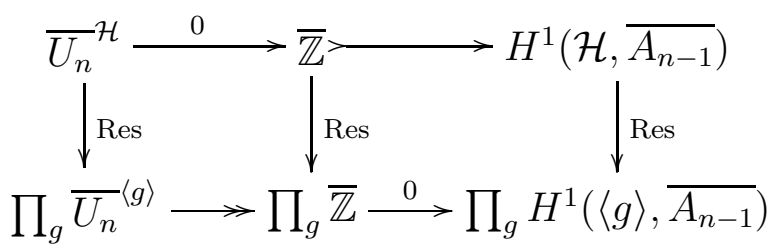

Here, the first 0 results from the fact that $\overline{U_{n}}$ is spanned by the orbit sums of the $\mathcal{H}$-orbits in $\{1, \ldots, n\}$, all of which have positive even length, while the second 0 is a consequence of the existence of $g$-fixed points, which implies surjectivity of ${\overline{U_{n}}}^{\langle g\rangle} \stackrel{\varepsilon}{\rightarrow} \overline{\mathbb{Z}}$ for all $g \in \mathcal{H}$. Thus, we conclude that the (nonzero) image of $\overline{\mathbb{Z}}$ in $H^{1}\left(\mathcal{H}, \overline{A_{n-1}}\right)$ does indeed belong to $\amalg^{1}\left(\mathcal{H}, \overline{A_{n-1}}\right)$, forcing this group to be non-trivial as required.

The actual construction of a suitable subgroup $\mathcal{H}$ is a simple matter. For example, we can take the group $\mathcal{H}=\langle\alpha, \beta\rangle$, with $\alpha=(1,2)(3,4) \cdots(n-3, n-2), \beta=$ $(1,2)(5,6) \cdots(n-1, n)$, that was used in the proof of Proposition 4.1(i) for $p=2, k \geq$ 3 ; see (4.3). This proves the assertion about $\mathrm{Sym}^{2} A_{n-1}$.

In order to show that $\bigwedge^{2} A_{n-1} \nsim A_{n-1}^{\otimes 2}$, it suffices to exhibit a subgroup $\mathcal{H} \leq \mathcal{S}_{n}$ such that $\amalg^{2}\left(\mathcal{H}, \bigwedge^{2} A_{n-1}\right) ¥ \amalg^{2}\left(\mathcal{H}, A_{n-1}^{\otimes 2}\right)$ In fact, the above $\mathcal{H}$ will do. For, by (11) and Proposition 4.1(i) (see also (4.3)), we know that $\amalg^{2}\left(\mathcal{H}, A_{n-1}^{\otimes 2}\right) \cong \amalg^{1}\left(\mathcal{H}, A_{n-1}\right)=$ $\mathbb{Z} / 2 \mathbb{Z}$. On the other hand, $\mathcal{H} \leq \operatorname{stab}_{\mathcal{S}_{n}}(\{n-1, n\})=\mathcal{S}_{n-2} \times \mathcal{S}_{2}$. Thus, it suffices to show that

$$
\amalg^{2}\left(\mathcal{H}, \bigwedge^{2} A_{n-1}\right)=\{0\}
$$

holds for every $\mathcal{H} \leq \mathcal{S}_{n-2} \times \mathcal{S}_{2}$. Consider the exact sequence

$$
0 \rightarrow \bigwedge^{2} A_{n-1} \rightarrow \bigwedge^{2} U_{n} \rightarrow A_{n-1} \rightarrow 0
$$

from Lemma 3.3(iii) and the resulting map $\amalg^{2}\left(\mathcal{H}, \bigwedge^{2} A_{n-1}\right) \rightarrow \amalg^{2}\left(\mathcal{H}, \bigwedge^{2} U_{n}\right)=$ $\{0\}$. Here, the last equality holds since $\bigwedge^{2} U_{n}$ is monomial; see Lemmas 3.3(i) and 4.4. Thus, in order to show that $\amalg^{2}\left(\mathcal{H}, \bigwedge^{2} A_{n-1}\right)=\{0\}$, it suffices to show that $H^{2}\left(\mathcal{H}, \bigwedge^{2} A_{n-1}\right) \rightarrow H^{2}\left(\mathcal{H}, \bigwedge^{2} U_{n}\right)$ is mono or, equivalently, that

$$
H^{1}\left(\mathcal{H}, \bigwedge^{2} U_{n}\right) \rightarrow H^{1}\left(\mathcal{H}, A_{n-1}\right) \text { is epi. }
$$

But, as $\mathcal{S}_{n-2} \times \mathcal{S}_{2}$-lattices (and hence as $\mathcal{H}$-lattices), $\bigwedge^{2} U_{n} \cong \mathbb{Z}_{\varphi} \uparrow_{\mathcal{S}_{n-2} \times \mathcal{S}_{2}}^{\mathcal{S}_{n}}$ contains $\mathbb{Z} \cdot u_{n} \wedge u_{n-1}$ as a direct summand, and $A_{n-1}$ contains $\mathbb{Z} \cdot\left(u_{n}-u_{n-1}\right)$ as a sublattice, with

$$
A_{n-1} / \mathbb{Z} \cdot\left(u_{n}-u_{n-1}\right) \cong \mathbb{Z}_{\mathcal{S}_{2}}^{\mathcal{S}_{n-2} \times \mathcal{S}_{2}} \text { a permutation lattice. }
$$


Moreover, $\mathbb{Z} \cdot u_{n} \wedge u_{n-1} \stackrel{\cong}{\longrightarrow} \cdot\left(u_{n}-u_{n-1}\right)$ under $\wedge^{2} U_{n} \rightarrow A_{n-1}$. Therefore, restricting $H^{1}\left(\mathcal{H}, \bigwedge^{2} U_{n}\right) \rightarrow H^{1}\left(\mathcal{H}, A_{n-1}\right)$ to the subgroup $H^{1}\left(\mathcal{H}, \mathbb{Z} \cdot u_{n} \wedge u_{n-1}\right)$, we obtain

$H^{1}\left(\mathcal{H}, \mathbb{Z} \cdot u_{n} \wedge u_{n-1}\right) \stackrel{\cong}{\longrightarrow} H^{1}\left(\mathcal{H}, \mathbb{Z} \cdot\left(u_{n}-u_{n-1}\right)\right) \rightarrow H^{1}\left(\mathcal{H}, A_{n-1}\right)$. This completes the proof.

\section{REFERENCES}

[1] A. Babakhanian, Cohomological Methods in Group Theory, Marcel Dekker, New York, 1972.

[2] E. Beneish, Induction theorems on the stable rationality of the center of the ring of generic matrices, Trans. Amer. Math. Soc. 350 (1998), 3571-3585.

[3] C. Bessenrodt and L. Le Bruyn, Stable rationality of certain $P G L_{n}$-quotients, Invent. math. 104 (1991), 179-199.

[4] N. Bourbaki, Algèbre, chap. 1-3, Hermann, Paris, 1970.

[5] K. S. Brown, Cohomology of Groups, Springer-Verlag, New York, 1982.

[6] J.-L. Colliot-Thélène and J.-J. Sansuc, La R-équivalence sur les tores, Ann. scient. Éc. Norm. Sup. 10 (1977), 175-230.

[7] J.-L. Colliot-Thélène and J.-J. Sansuc, Principal homogeneous spaces under flasque tori: Applications, J. Algebra 106 (1987), 148-205.

[8] A. Cortella and B. Kunyavskiǔ, Rationality problems for generic tori in simple groups, preprint, 1999.

[9] W. Fulton and J. Harris, Representation Theory, A First Course, Springer-Verlag, New York, 1991.

[10] E. Formanek, The center of the ring of $3 \times 3$ generic matrices, Linear and Multilinear Alg. 7 (1979), 203-212.

[11] E. Formanek, The center of the ring of $4 \times 4$ generic matrices, J. Algebra 62 (1980), 304-319.

[12] M. Hajja and M.-C. Kang, Three-dimensional purely monomial group actions, J. Algebra 170 (1994), 805-860.

[13] P. H. Kropholler and B. Moselle, A family of crystallographic groups with 2-torsion in $K_{0}$ of the rational group algebra, Proc. Edinburgh Math. Soc. 34 (1991), 325-331.

[14] I. J. Leary, A torsion projective class for a group algebra, Bull. London Math. Soc. (to appear).

[15] I. J. Leary, The Euler class of a Poincaré duality group, preprint, University of Southampton, May 1998.

[16] L. Le Bruyn, Centers of generic division algebras: The rationality problem 1965-1990, Israel J. Math. 76 (1991), 97-111.

[17] H. W. Lenstra, Rational functions invariant under a finite abelian group, Invent. math. 25 (1974), 299-325.

[18] M. Lorenz, Picard groups of multiplicative invariants, Comment. Math. Helv. 72 (1997), 389399.

[19] C. Procesi, Non-commutative affine rings, Atti Accad. Naz. Lincei, VIII. Ser., v. VIII, fo. 6 (1967), 239-255.

[20] D. Saltman, unpublished letter, December 1986.

[21] R. G. Swan, Noether's problem in Galois theory, In: B. Srinivasan and J. Sally (eds.), Emmy Noether in Bryn Mawr, Springer-Verlag, Berlin-Heidelberg-New York, 1983, pp. 21-40. 
Department of Pure Mathematics, University of Waterloo, Waterloo, On, Canada, N2L 3G1

E-mail address: nlemire@math.uwaterloo.ca

Department of Mathematics, Temple University, Philadelphia, PA 19122-6094

E-mail address: lorenz@math.temple.edu 